\title{
TORRES DE AGUA EN LIMA. CONDICIÓN DE PATRIMONIO UTILITARIO DEL SIGLO XX $X^{(*)}$
}

\author{
WATER TOWERS IN LIMA. \\ STATE OF UTILITARIAN HERITAGE OF THE 2OTH CENTURY
}

ISRAEL LEANDRO FLORES ${ }^{(\star \star)}$

Fecha de recepción: 14 de setiembre de 2015

Fecha de aprobación: 14 de octubre de 2015

\begin{abstract}
RESUMEN
La ciudad genera hitos o monumentos si y solo si es capaz de inventarlos o reconocerlos. Sea cual fuere el caso, la valoración de un bien o de un artificio va de la mano con el auto reconocimiento como persona y como colectivo. El reconocimiento de parte de la sociedad debe darse a través de sí misma y de su contexto físico y mental. Es un ejercicio de autoafirmación. El reconocer su patrimonio es un ejercicio pleno de la autoestima y la razón colectiva. Pero, ¿qué pasa cuando la sociedad no sabe qué bienes posee? ¿Se podría hablar de un patrimonio no reconocido, o se debería hablar de un patrimonio no valorado? El presente texto pretende argumentar las condiciones que debería cumplir en este caso el testimonio del crecimiento de la ciudad, como lo son las torres de agua en Lima, así como el porqué de su aceptación como memoria colectiva y razón valorativa.
\end{abstract}

\section{PALABRAS CLAVE}

Dasein, patrimonio, significancia

\begin{abstract}
The city generates landmarks or monuments only if it is able of inventing or recognize them. Either way, the valoration of an asset or an artifact goes in hand with the assumption as a human being and as a collective. Society as such requires to recognize through itself and its reality, in other words, its physical context and mental context. As a self-assertion. Knowing its heritage is a plain exercise of self-esteem and collective reason. But, what happen when society doesn't know what assets they have? Can we talk about an unrecognized heritage? Or maybe a diminished heritage? This work pretends to make a statement about the conditions that must comply the testimony of the city's growth, as are the Water Towers in Lima, and as the Water Towers in Lima and the reasons of its acceptance as collective memory and valorative reason.
\end{abstract}

\section{KEYWORDS}

Dasein, heritage, significance

(*) El presente artículo "Torres de agua en Lima. Condición de patrimonio utilitario del siglo XX" tiene como base la investigación titulada "Torres de aqua en Lima", desarrollada en el Taller de investigación 2006-1, dentro del marco académico de la Facultad de Arquitectura y Urbanismo de la Pontificia Universidad Católica del Perú, curso de Seminario de Investigación, el cual fue dirigido por el Prof. Dr. Wiley Ludeña Urquizo.(**) Arquitecto por la Pontificia Universidad Católica del Perú. Trabajó como responsable de campo y gabinete del equipo local en el Proyecto de Reconstrucción y Ordenamiento del Centro Urbano de Huaytará (Fondo Italo Peruano-Universidad de Roma Tres). Trabajó para el Coloquio Internacional Creaciones Geográficas, Realidades Geopolíticas, Fronteras y Movilidad con la Cooperación Francesa (Universidad de París 8, COARTERRA, Retina Internacional). Contacto: tempus182@gmail.com 


\section{Definición Patrimonial de las Torres de Agua}

La definición de patrimonio que en este texto se tiene como pertinente, dentro de las tantas existentes, es la siguiente: “...patrimonio viene del latín patrimonium; es aquello que proviene de los padres. Según el diccionario, patrimonio son los bienes que poseemos, o los bienes que hemos heredado de nuestros ascendientes. Lógicamente patrimonio es también todo lo que traspasamos en herencia..." (Ballart \& Juan-Tresserras, 2001, p. 11). Es también argumento fuerte el determinar las tres características esenciales: la temporalidad, la significación y la relatividad (Hayakawa, 2012, p. 4). En otra referencia:

Todo patrimonio se encuentra acotado en el espacio y en el tiempo. En ese sentido se podría hablar de distintos patrimonios.... Pero además el patrimonio no es inmutable, varía con el tiempo.... Todo patrimonio es un conjunto muy variado de elementos heredados, de distinta naturaleza. Heredados por personas, grupos sociales o la humanidad en su conjunto. Pero puede no existir la conciencia por parte de los herederos de la importancia de los bienes que reciben; es decir, dichos bienes nada significan para ellos. No existe vinculación alguna entre el bien heredado y los valores en que creen los herederos... En tal caso, el patrimonio puede desaparecer, transformarse... sin que nadie se dé cuenta... Pero el mismo concepto de significación, como implica valores y por tanto mundos de vida diferentes, es un concepto relativo en sí mismo. Depende de los puntos de vista de los diferentes actores involucrados.... (Hayakawa, 2012, p. 5)

En el caso de un 'bien', este puede ser material o inmaterial, según la interesante acepción relacionada con el Derecho ( $6^{\circ}$ acepción). Es decir, entrando al campo metafísico, este puede trascender a la materia si en la construcción mental individual o colectiva se le considera valorativamente. Así, al valor del bien se le construye en el tiempo, y este, si trasciende, es porque es resiliente. $Y$ si es resiliente, es porque factores externos o internos permiten su supervivencia. Esta supervivencia es la heredad, y uno hereda lo que dejaron los ascendientes. Ahora, se debe considerar que hay resiliencia, en contraste con la obsolescencia: si bien es cierto que han permanecido, esto no se debe precisamente a su aceptación como bien; es precisamente lo contrario de esto.

La permanencia de las torres y su bajo índice de demolición, salvo algunos casos y el de la Unidad Vecinal 3, el cual se discutirá más adelante, se debe al elevado costo directo e indirecto de, verbigracia, demolerlas. Esta actitud, como típica política inactiva y contemplativa, hace notar una doble entrada de parsimonia y ceguera de parte de la sociedad: no se hace nada porque no importa y no importa porque no se hace nada.

En cuanto al segundo caso, la 'temporalidad', si el tiempo es lo que moldea la permanencia física y metafísica del argumento patrimonial, el espacio es lo que le da materialidad. Es en esta dualidad espacio-temporalidad que la ignominia de entenderse como ser sale a la luz.

Observemos el argumento de Martin Heidegger en su seminal obra Sein und Zeit, construido sobre la base de un concepto fundamental de Edmund Husserl, el Dasein. En esencia, el Dasein es una composición compleja pero simple a la vez. Si se parte en dos el compuesto (asumiendo como cierto el argumento de palabra compuesta de Jorge Eduardo Rivera) se obtiene dos lecturas: el das (artículo neutro) y el ein (como uno), lo cual da como resultado 'lo uno', y la otra lectura da (en segunda acepción el ahora) y el sein (en primera acepción el ser), que resulta en el 'ser ahora'

Es entonces lógico asumir dos aspectos: la atención de tener un 'lo uno', como lo único, como lo importante, y la otra parte, el 'ser ahora', como lo trascendente en lo temporal. Ambas lecturas, asumiéndolas como válidas, sintetizan los dos sentidos del tema trascendental. Lo más importante es que en la combinación de ambas sustancias está inmanente el 'ser ahí' (Heidegger, 1927/2003). 
El éxito de entender esto es la rotura cartesiana y, por ende, posclásica, de la división racional entre el sujeto y el objeto. Heidegger plantea la fusión de ambos en la malla espacio-temporal. Debe considerarse que el término exacto es "ser arrojado-eyectado a la realidad" (Heidegger, 1927/2003, p. 52). Entonces, para ser completo en la realidad como existencia temporal, uno tiene que ser consciente de su papel en el contexto. A estas luces, según Heidegger, el Dasein no está completo mientras no acepta su papel en el contexto espacio-temporal.

Es en ese sentido que la sociedad no reconoce que es de valor por la temporalidad, dado que la sociedad misma, entendida como suma de individuos con argumentos comunes, no se reconoce en el contexto, es decir, en su realidad. La sociedad no ha terminado, ni siquiera empezado, a formar su Dasein. Al no entender su contexto, ergo, su tiempo, no puede reconocer el valor temporal.

Ello deriva al siguiente caso, la significación. Entre las acepciones de la palabra, encontramos las siguientes: "(Del lat. significatǐo, -ōnis). 1. f. Acción y efecto de significar. 2. f. Sentido de una palabra o frase. 3. f. Objeto que se significa. 4. f. Importancia en cualquier orden" (Significación, 2005, p.1401). La palabra, en sus cuatro acepciones, encierra trascendencia respecto al objeto y la proyección que este tiene al sujeto, y también que el sujeto proyecta hacia el objeto. Esta doble proyección es clave para entender como un hecho o ente puede tener significado, y posteriormente valor en el imaginario individual o colectivo: uno se reconoce en lo otro y viceversa. En un primer momento requiere un rol activo: a) la síntesis del sujeto b) proyectada a un objeto, c) y, por último, el rol en el ente. Estas partes del todo suman un ser complejo en la metafísica.

Según la transcripción de una conferencia dada por Heidegger en Brême, en 1930:

Lo verdadero, ya sea una cosa verdadera o una proposición verdadera, es aquello que concuerda, lo concordante. Ser verdadero y verdad significan aquí concordar en un doble sentido: por un lado como concordancia de una cosa con lo que previamente se entiende por ella, y, por otro, como coincidencia de lo dicho en el enunciado con la cosa. Este doble carácter del concordar se pone de manifiesto en la tradicional definición de la verdad: veritas est adaequatio rei et intellectus. Esto puede significar que la verdad es la adecuación de la cosa al conocimiento.

Pero también puede querer decir que la verdad es la adecuación del conocimiento a la cosa. Ciertamente, por lo general se suele presentar la definición esencial ya citada bajo la formulación que dice: veritas est adaequatio intellectus ad rem. Pero la verdad así entendida, la verdad de la proposición, sólo es posible sobre el fundamento de la verdad de la cosa, la adaequatio rei ad intellectum. Ambos conceptos esenciales de la veritas significan siempre un regirse de acuerdo con o conformarse a y, por ende, piensan la verdad como conformidad o rectitud. (...) (Heidegger, 2009, p. 6)

De la densidad de la expresión de Heidegger es vital rescatar, antes que el argumento, una palabra útil para definir su enlace de verdad y verdadero, característica magnífica de la significancia: concordancia. En sentido figurado, encuentro de corazones. La concordia es tener un solo corazón o un corazón compartido. Aquí la esencia de la significancia: hacer un solo corazón con el ente, el bien hacia el contexto.

Es en ese compartir corazones o, más explícitamente, voluntades, que encontramos la razón dialéctica de sujeto y objeto. Si argumentamos la esencia kantiana de sujeto-objeto, en contraposición a la partida heideggeriana, lo significante para el individuo o colectivo se demuestra en la identificación del artificio, del hecho producto de la intervención del sujeto en el contexto. Es decir, la significancia viene a ser un síntoma del Dasein colectivo estructurado, que permanece en el contexto vivido. Se recuerda el término como proyección hacia el contexto, hacia la realidad. Al no existir, por lo menos desde un inicio el Dasein autónomo, aislado, es fácil inferir que cualquier demostración en el contexto es parte de lo importante y significativo si 
y solo si trasciende en las construcciones reales y abstractas. Aquí el enlace con la temporalidad del patrimonio.

Como se observó, la temporalidad es el plano trascendente compartido con el contexto real. Así también, la significancia es lo que mantendrá unidas estas dos partes. Si el Dasein es la conexión proyectada, la significancia es el aglomerante mental y real que las mantiene unidas y compartidas.

Otra situación del mismo texto es la cita de 'verdad' "que se define dualmente ya sea como la adecuación de la cosa (ente valorativo) al conocimiento y también la adecuación del conocimiento a la cosa" (Heidegger, 2009, p. 6).

La importancia de esta cita dentro de nuestro argumento se encuentra en la relación de la significancia con la verdad. La palabra 'significancia' aquí es un anglicismo: "1. n. the quality of being important. 2 . $n$. the quality of having notable worth or influence $3 . n$. the meaning of something." (Significance, 2014). Esto argumenta nuestro caso, pues tiene los componentes de cualidad. Esta cualidad no es otra sino la dada por el ente.

Por otro lado, en las ciencias estadísticas, el valor de significancia, simplemente la significancia para nuestro caso, mide el valor de verdad de una proposición sometida a un análisis lógico. Esto se deriva del nivel de probabilidad de que haya venido de un azar. Se debe tomar en cuenta que esto no verifica la verdad absoluta de la premisa, sino la confianza del bajo error de las pruebas. Es decir, siguiendo los postulados normados, a más pruebas se debe obtener una postulación de por lo menos $95 \%$ de confianza. Recién a partir de allí es posible valorar el grado de no-azar de la postulación.

Esto resulta importante para el argumento de significancia respecto al valor patrimonial. Si se postula la significancia como búsqueda de la mayor verdad o el mayor valor estadístico de la verdad, esta es inherente al ser humano, al ente del que se requiere un arrojo, un Dasein.

Es en esta búsqueda de la verdad (significación), en que el colectivo o el individuo que lo colectivo busca o debe buscar en el tiempo (temporalidad), donde entra a tallar el último de los argumentos: la relatividad.

Reconocer no implica valorar. ¿Cómo se logra entender el valor de algo?

Aquí se mantendrá este algo en lo neutro, en el objeto/sujeto. En este tercer paso, la relatividad, es necesario analizar también el tiempo. Se dice que cada quién tiene un valor de verdad intrínseco, que cada quién tiene su forma de ver la realidad. Estos postulados populares tienen una gran limitación: el dejar de pensar en el colectivo, el excluir lo propio de lo otro. En un sentido, es generar una otredad ajena, es excluir y autoexcluirse del colectivo. El ente patrimonial, que es el hecho de la valoración de algo como propio, parte del desarrollo de consciencia colectiva, y la consciencia colectiva no se logra sino en el tiempo. Asimismo, esta situación colectiva no se logra sin ser conscientes, sin generar una identificación de lo propio. La relatividad entra a tallar en tanto el Dasein-colectivo es consciente de los cambios a lo largo del tiempo. Para lograrlo es crucial eliminar la otredad desde el punto de vista de cosmovisión. ¿Es esto posible? Es posible si y solo si nos debemos y cambiamos de otredad a la alteridad. Se debe resignificar el término.

La definición de alteridad es la siguiente: "(Del lat. alteritas) 1. f Condición de ser otro." (Alteridad, 2005, p. 84). Esta definición es curiosamente la misma, según la Real Academia Española (2005), que la de 'otredad'. Sin embargo, habría que establecer el argumento ético. Explicar los dos conceptos de alteridad trae a colación una serie de puntos interesantes en relación al tema en cuestión. 
La primera definición indica que es necesario establecer la alteridad como una necesidad vital: "su pertenencia a una sociedad y a una raza, junto al sentimiento" (Vázquez Fernández, 2014, p. 76). La segunda plantea que es una razón de dependencia y filiación: "esta matriz crítica y resituacionista comienza a tomar forma alrededor de una cuestión fundamental: si el ser sólo se puede afirmar en la medida en que es conocido, ¿cuál es el ser del otro?" (Vázquez Fernández, 2014, p. 77)

El hecho innegable es que la alteridad es necesaria para buscar la identidad de uno mismo respecto de un universo construido también por uno mismo. Definitivamente no puede haber el uno sin el otro, debido a la dialéctica inmanente. Ahora, el hecho de identificarse como uno, como ser, el ahora entregarse al Dasein completo, ya de por sí marca una diferencia fundamental en el pensamiento. El otro ya no se forma por antonimia, sino que se forma como compartir el tiempo-espacio. El otro pre-Dasein se forma por un devenir natural reflexivo, no por un entendimiento de la realidad total; se hace por el individualismo, el ser reconocido por uno solo, por uno mismo; nace porque el contexto-temporal-físico me hace dependiente de la realidad y esta está inundada de otros.

Esta realidad hace que el otro-Dasein sea más activo. El sentido de acción del otro hace nacer, para nuestros fines, la colectividad.

Esta colectividad, por lo tanto, tiene que nacer de un trauma, que es el reconocerse en el contexto, ya no en uno mismo, como ente o en otro por reflexión. La ventaja de entenderse en el contexto físico-temporal es que permite al Dasein-colectivo precisamente compartir la realidad; si se comparte la realidad, se establece desde ya un universo común e independiente a la vez.

El Dasein es el único que tiene la capacidad, o podríamos decir facultad, de hacerse la pregunta del ser. Por lo mismo, el otro-Dasein debe también compartir esas preguntas; al compartirlas se genera el ser colectivo, es decir, el Dasein-colectivo. Lo importante de entender de esta argumentación es que finalmente la relatividad se origina en ello, en que la suma de Dasein, otro-Dasein y Dasein-colectivo son entes totalmente arrojados a su contexto; dado que el contexto cambia, si cambia, los Dasein completos también van a cambiar.

En términos de crítica sociológica sobre el actual estado de la colectividad, se puede afirmar que el colectivo no existiría sin el reconocimiento de uno, del otro y de todos. Por más que parezca evidente e incluso absurdamente lógico, es importante entenderlo a la luz del ser-relativo. El contexto que cambia debe cambiarse con el todo, no por la suma de individuales. El gran problema actual es que cuando el contexto físico temporal cambia, el individuo no cambia como el otro, dado que todavía no se llega al otro-Dasein y menos al Dasein-colectivo. El verdadero razonamiento del Dasein-colectivo provoca la relatividad. Si uno es con el otro y el colectivo, también es por uno mismo. Los agentes o artefactos cambiantes en todo el tiempo-espacio precisamente no se desvaloran ellos mismos, sino la colectividad que no se reconoce a sí misma.

Cabe precisar que, si el bien patrimonial no es inmutable, se carga también a su ilógica de pensar un aparato que no se vea afectado por el tiempo, no por una trascendencia ganada por derecho divino, sino porque el Dasein-colectivo es capaz de reconocerse cada vez que se cambia en el contexto. Lo relativo, en el argumento patrimonial, pasa entonces por compartir otra vez esa concordia.

El hecho cambiante del ente patrimonial es absoluta demostración de que la caracterización de lo proyectado hacia la realidad es menester del Dasein. Entonces, si se plantea algo relevante al colectivo otra vez, entendido como arrojado al contexto, el ente patrimonial debería ser parte de ese universo. 
La aceptación de algún ente o no por parte de esto es clave para transversalizar todo: la temporalidad, la significación y la relatividad. Se entiende entonces que la realidad patrimonial, al tener estos componentes, depende de la identificación del Dasein con la realidad. De allí se infiere que cualquier hecho que tenga que ver con el ente patrimonial y la definición de su valor para el colectivo tiene que ver con una consciencia del contexto. La propuesta de este texto consiste entonces, en primer lugar, en conocer un ente patrimonial; en segundo lugar, demostrar su reconocimiento con el $\mathrm{Da}$ sein-colectivo; y, por último, con la condición de patrimonio arquitectónico utilitario.

\section{Argumento Urbano-Patrimonial de las Torres de Agua}

Dentro de la práctica usual arquitectónica se nomina, incluso se corrige, el concepto de ciudad. Las visiones de ciudad han cambiado a lo largo de la historia. Una primera definición describe a la ciudad como "las amalgamas de edificios y personas (...) es el mejor memorial de nuestras luchas y continuidades en el tiempo y el espacio" (Kostof, 1991, p. 14). Otra la define como "la manifestación física de nuestras interacciones" (West, 2015). Ambas, si bien similares, esconden una diferencia fundamental. En el caso de Kostof, este trata de definir un porqué. En el caso de West, este se limita, respetuosamente, a un qué. Se afirma que Kostof refiere al porqué dado que incluye el factor tiempo; este factor de morfología urbana, el argumento einsteiniano malla espacio-temporal, hace que se tenga bastante a bien la carga de la historia. Es el tiempo usado como herramienta. En el caso de West, el tiempo es una excusa, un 'catalizador' visual de los problemas. En una se ve la explicación patrimonial y en otra la urbana. En este caso tomaremos la segunda.

El interés en definir la importancia de las torres de agua reside precisamente en que son testimonio del crecimiento de la ciudad, absoluta e irónicamente anónimo. Se dividirá los argumentos en cuatro secciones: definición de las torres de agua, definición patrimonial, definición urbana y argumento sintetizador. En ese sentido, se argumentará la condición de patrimonio arquitectónico utilitario de las torres de agua.

\section{Definición de las Torres de Agua}

Según el Diccionario de la Real Academia de la Lengua Española, la palabra 'torre'tiene ocho acepciones directas. Las siguientes son las tres primeras:

1. f. Edificio fuerte, más alto que ancho, y que sirve para defenderse de los enemigos desde él, o para defender una ciudad o plaza.

2. f. Edificio más alto que ancho y que en las iglesias sirve para colocar las campanas, y en las casas para esparcimiento de la vista y para adorno.

3. f. Edificio de mucha más altura que superficie. (2005, p. 1493)

Sea el caso, todos devienen de la palabra 'turris'. Esta palabra, según se indica en Online Etymology Dictionary, tiene tres acepciones, ya sea como verbo o como sustantivo.

tower (v.) c. 1400, "rise high" (implied in towered); see tower (n.). Also, of hawks, "to fly high so as to swoop down on prey" (1590s). Related: Towering.

tower (n.1) Old English torr "tower, watchtower," from Latin turris "a tower, citadel, high structure" (also source of Old French tor, 11c.; Spanish, Italian torre "tower"), possibly from a pre-Indo-European Mediterranean language. Meaning "lofty pile or mass" is recorded from mid-14c. Also borrowed separately 13c. as tour, from Old French tur; the modern spelling (1520s) represents a merger of the two forms.

tower (n.2)"one who tows," 1610s, agent noun from tow (v.). ("Tower", 2015) 
En el argot técnico se les denomina 'reservorios elevados de agua', y en el saber popular, según una encuesta limitada realizada hace ocho años, el nombre más empleado era 'tanque' (Leandro Flores, 2006). Este dato resulta interesante pues permite observar la extrapolación de términos que la gente asume en distintas escalas. La principal razón de ello es la cercanía que la población en general tiene a su referencia vivencial: en términos técnicos reales se llama 'tinaco', pero el popular es 'tanque', aquel que se coloca en las azoteas para generar mayor presión de agua.

El funcionamiento es matriz de la gravedad: el agua, que debería llegar a ciertas partes de la ciudad, no llega con la suficiente presión. Por ello se sube el contenedor de agua a una altura calculada para abastecer de presión necesaria; es una regla directamente proporcional: a mayor altura, mayor presión. También entran a tallar otros términos como 'columna de agua,' 'ecuación de Bernoulli' y'derivaciones hiperestáticas', pero en sentido práctico la regla se mantiene: a más altura, más fuerza de caída y más presión final. En términos normativos georeferenciados, resulta necesario recurrir a Sedapal (Servicio de Agua Potable y Alcantarillado de Lima), institución que nomina básicamente el término 'reservorios' para todos los casos. En el caso aquí tratado hay tres referentes: reservorio enterrado, es decir, que está debajo de la cota de tierra; reservorio apoyado, que está en la cota de tierra; y reservorio elevado, que está por encima de la cota de tierra. Al respecto, Sedapal indica que en Lima existen 849 reservorios de agua. De estos, 171 son del tipo tanque de agua (incluyendo las particulares), es decir, el 20,1\%.

De esos 171, se estima, dado que Sedapal no tiene un registro unificado actualizado del estado de funcionamiento de los mismos, que 25 están en operación continua (Leandro Flores, 2006). La descentralización del registro de Sedapal, que desde hace más de 30 años fragmentó sus responsabilidades en zonas para manejar mejor la cobertura, hace cada vez más difícil su actualización.

Por la propia condición de hito de las torres de agua, su condición de altura, y su distancia y ubicación dentro del tejido y las condiciones históricas, las cuales discutiremos más adelante, consideramos aquí que la esencia de las torres es el hecho de que tenían que ser necesariamente más altas que las casas que alimentaban. Si se calcula el radio de acción de las torres de agua, se debe tener en cuenta que su proceso constructivo no es para nada rápido. La edificación de una torre de agua de tamaño promedio (35 metros de altura) puede demorar, hasta su puesta en función y operatividad, hasta 18 meses, y es uno de los metros cuadrados techados más costosos de construir. Ello se debe a que su fuste de 35 metros de altura es ocho veces mayor que el promedio de 1.8 pisos de altura, alrededor de 4.5 metros, que en el PLAM 2035 se estima para Lima.

Las torres de agua se ubican, como puede verse en el la Figura 3, en un gran porcentaje en parques o plazas (72\%), disposición reglamentada en las primeras leyes de ocupación de viviendas. Ya de por sí, dentro del hecho estructuralista del reconocimiento Lyncheano de hecho mojonesco o de hito, marca distancia de cualquier accidente causal de una ciudad de facto. Ello tiene una condición dentro del tejido, la cual se analizará en la siguiente sección.

\section{Desarrollo Físico de las Torres de Agua}

Para conocer las torres de agua, básicamente se estableció el componente taxonómico de las mismas. Desde la investigación primera (Leandro Flores, 2006) han surgido actualizaciones de base tanto a nivel conceptual como a nivel metodológico. En el caso del nivel metodológico, para poder clasificarlas, se tomó en cuenta su geometría de base. Se planteo considerar también la ubicación, pero ese factor fue descartado debido a que la gran mayoría (89\%) se encontraba en los parques de base; no tenía argumento de desviación estadística lo suficientemente fuerte como para ser considerado. La altura también se consideró como posible factor, pero, al igual que con la ubicación, todas superaban los 30 metros de altura (valor estimado para muestra de 
devenir Vol. 2, Nº4, JULIO-DICIEMBRE 2015, PP. $73-90$ - EstudIOS | ISSN 2312-7570

UNIVERSIDAD NACIONAL DE INGENIERÍA, LIMA

Figura 1.Tipología comparada de las torres de agua, Rumania-Lima Fuente: Elaboración Propia, 2015.
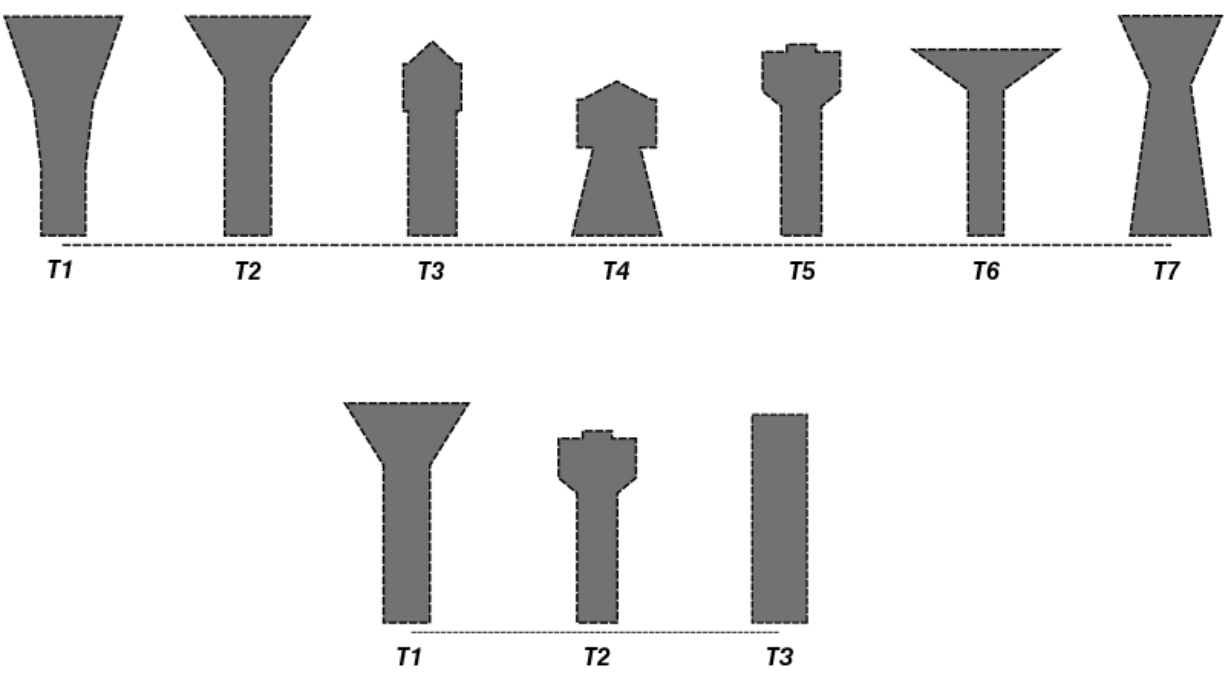

50 torres). Se evaluó también tomar en cuenta el material, pero la gran mayoría (95\%) eran de concreto armado. En cuanto a una clasificación según su antigüedad, esta se descartó pues, desde el inicio del estudio hasta la actualidad, no ha habido información concluyente ni oficial estructural de las fechas de construcción de las mismas.

Para la clasificación geométrica, se reunió todas las volumetrías posibles encontradas en las 171 torres estudiadas. Los resultados de la clasificación de los grandes grupos son los siguientes:

Prismática pura. Esta geometría se caracteriza por presentar en casi la totalidad de su fuste una geometría uniforme. Es llena; es decir, está conformada por superficies conectadas en todo su recorrido. Tiende a parecer sólido clásico.

Fungiforme. Esta geometría, como su nombre lo indica, tiene forma de seta u hongo. Se toma la clasificación típica de las mismas. Así presenta base, que generalmente contiene los controles y los accesos desde el nivel de calle; el fuste, que es el soporte estructural; $y$ el reservorio propiamente dicho. Casi siempre el reservorio supera en envergadura al fuste. En varios casos, el fuste puede también ser sólido o puede ser hueco; es decir, está conformado por un sistema aporticado de vigas y columnas de concreto armado.

Prismática compuesta. Esta geometría es irregular, ya sea en su fuste o en su reservorio. En la clasificación hecha, normalmente, es una combinación de las dos primeras. Esta suele llamar más la atención dado su impacto visual en el tejido regular en que se encuentra.

Para dar un argumento de clasificación más gráfico se presenta como referencia el estudio llevado a cabo por Cercleaux, Merciu \& Peptenatu (2014), una de las primeras publicaciones que logró establecer una matriz geométrica de base para las torres de agua en Rumania.

En la Figura 1 puede verse en la primera línea los resultados de la investigación citada en Rumania, y en la segunda línea, los resultados de la investigación hecha en Lima. La principal diferencia es la cantidad de clases determinadas por la investigación. En 


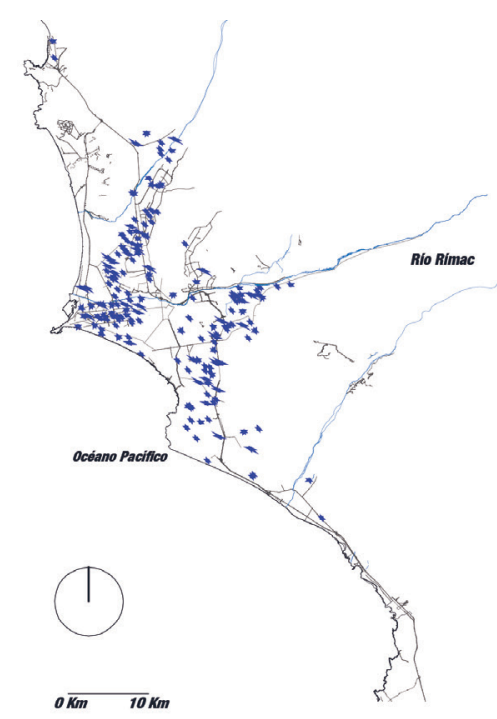

Mapeo según Torres de Agua

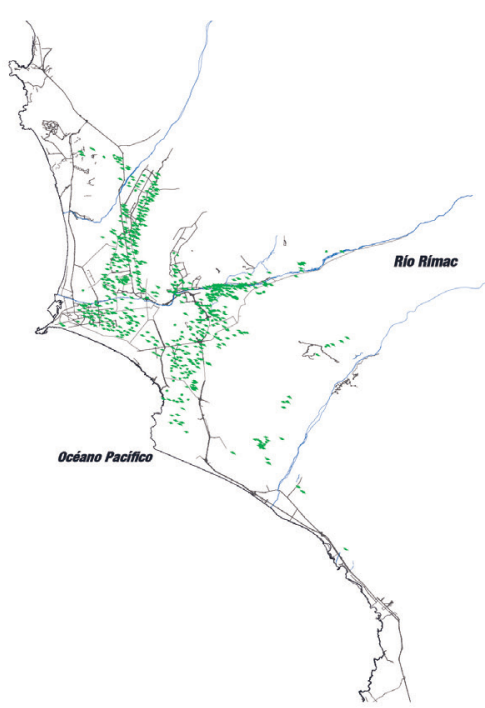

Mapeo según pozos

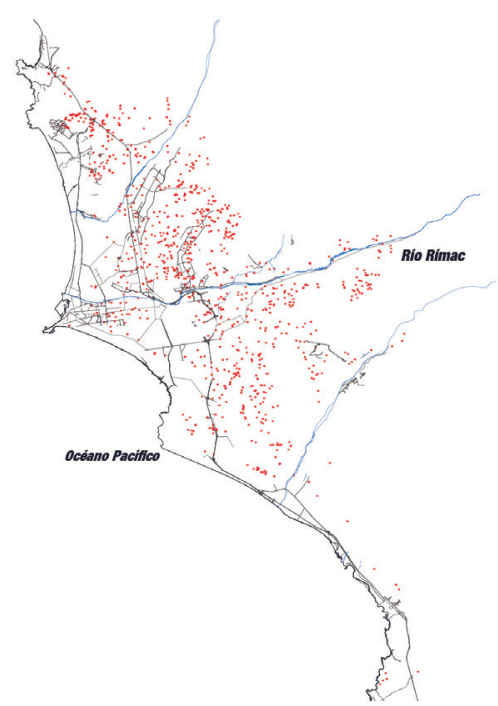

Mapeo según reservorios totales el caso de las torres de Rumania se establecen siete clasificaciones; sin embargo, la publicación no menciona ni grafica los tipos encontrados en su totalidad. En el caso de Lima, se llegó a reconocer tres tipos importantes, ya descritos según su geometría.

Para la clasificación de tal geometría se desglosó: un código de reconocimiento, propiedad, distrito de ubicación, coordenadas norte y este, distancia desde un punto base (Plaza de Armas) y la altura relativa al nivel del mar. La fuente oficial, una vez clasificada, se refiere al plano de Sedapal, obtenido en abril de 2015. Este mapeo de Sedapal registraba todos los reservorios de agua de Lima sin distinguir el tipo, así que se tuvo que limpiar la data levantada también desde campo.

Para el proceso de levantamiento se usaron imágenes satelitales disponibles en fuentes públicas como Google Earth, así como relevaciones realizadas con instrumentos de geolocalización propios, como se muestra en la Tabla 1. Para la instrumentalización de los datos se empleó una serie de cartas base, como la de crecimiento por barrios, áreas verdes y vías. Ello daría las luces sobre la correlación de las torres de agua según las diferentes capas del contexto metropolitano. En el caso de las correlaciones directas, se establecieron los mapeos de las torres de agua, que se compararon con la base de datos de Sedapal de pozos y a reservorios de todos los tipos.

A partir de esos datos se pueden inferir las siguientes conclusiones:

- La altura del distrito es inversamente proporcional a la cantidad de torres de agua. En efecto, según la data mostrada, los distritos que presentan la mayor cantidad de torres de agua son los que cuentan con menores estribaciones andinas. Mientras el mapeo se acerca a la zona Este de la ciudad, menor es la concentración de torres de agua.

- Lima Norte concentra la mayor cantidad de torres de agua. Esto puede correlacionarse a partir de la carta de crecimiento formal versus informal. Como se puede ver en la Figura 2, los distritos más poblados, como Los Olivos, Comas y San Martín de Porres, concentran 39 torres de agua, que representan el 23\% del total. Estos sectores en su mayoría fueron consecuencia del crecimiento espontáneo que tuvo lugar desde inicios del siglo XX.
Figura 2. Mapeo de los reservorios en Lima Metropolitana

Fuente: Elaboración propia, 2015. 
devenir Vol. 2, N4, JULIO-DICIEMBRE 2015, PP. 73-90 - EstudIOS | ISSN 2312-7570

UNIVERSIDAD NACIONAL DE INGENIERÍA, LIMA

Tabla 1. Listado de Torres de agua. Se observan los indicadores.

Fuente: Elaboración propia, 2015.

\begin{tabular}{|c|c|c|c|c|c|c|}
\hline CÓDIGO & PROPIEDAD & DISTRITO & $\begin{array}{l}\text { COORDENADA } \\
\text { NORTE }\end{array}$ & $\begin{array}{l}\text { COORDENADA } \\
\text { ESTE }\end{array}$ & $\begin{array}{c}\text { DISTANCIA DESDE LA PLAZA } \\
\text { DE ARMAS }\end{array}$ & ALTURA RELATIVA \\
\hline 001 & SEDAPAL & EL AGUSTINO & 8666194 & 281052 & $2.55 \mathrm{~km}$ & 178 m.n.s.m. \\
\hline 002 & PARTICULAR & EL AGUSTINO & 8668796 & 281409 & $2.73 \mathrm{~km}$ & 190 m.n.s.m. \\
\hline 003 & SEDAPAL & LA VICTORIA & 8664650 & 280020 & $3.17 \mathrm{~km}$ & 148 m.n.s.m. \\
\hline 004 & SEDAPAL & CERCADO & 8668640 & 275009 & $4.10 \mathrm{~km}$ & 95 m.n.s.m. \\
\hline 005 & SEDAPAL & CERCADO & 8667761 & 274767 & $4.18 \mathrm{~km}$ & 97 m.n.s.m. \\
\hline 006 & SEDAPAL & EL CERCADO & 8664650 & 280020 & $4.35 \mathrm{~km}$ & 93 m.n.s.m. \\
\hline 007 & SEDAPAL & CERCADO & 8665896 & 274789 & $4.51 \mathrm{~km}$ & 92 m.n.s.m. \\
\hline 008 & SEDAPAL & EL CERCADO & 8665961 & 273825 & $5.38 \mathrm{~km}$ & 79 m.n.s.m. \\
\hline 009 & SEDAPAL & CERCADO & 8668497 & 273587 & $5.43 \mathrm{~km}$ & 74 m.n.s.m. \\
\hline 010 & SEDAPAL & LA VICTORIA & 8663646 & 282574 & $5.40 \mathrm{~km}$ & 179 m.n.s.m. \\
\hline 011 & SEDAPAL & EL CERCADO & 8665859 & 273799 & $5.50 \mathrm{~km}$ & 78 m.n.s.m. \\
\hline 012 & SEDAPAL & ATE & 8665143 & 284003 & $5.64 \mathrm{~km}$ & 213 m.n.s.m. \\
\hline 013 & SEDAPAL & CALLAO & 8667217 & 273184 & $5.78 \mathrm{~km}$ & 69 m.n.s.m. \\
\hline 014 & SEDAPAL & CARMEN DE LA LEGUA & 8667864 & 273157 & $5.79 \mathrm{~km}$ & 71 m.n.s.m. \\
\hline 015 & SEDAPAL & EL CERCADO & 8666578 & 273191 & $5.86 \mathrm{~km}$ & 71 m.n.s.m. \\
\hline 016 & PARTICULAR & BELLAVISTA & 8666303 & 272649 & $6.43 \mathrm{~km}$ & 65 m.n.s.m. \\
\hline 017 & SEDAPAL & ATE & 8664198 & 284448 & $6.50 \mathrm{~km}$ & 207 m.n.s.m. \\
\hline 018 & SEDAPAL & ATE & 8665793 & 285190 & $6.51 \mathrm{~km}$ & 233 m.n.s.m. \\
\hline 019 & SEDAPAL & ATE & 8663863 & 284334 & $6.57 \mathrm{~km}$ & 202 m.n.s.m. \\
\hline 020 & SEDAPAL & BELLAVISTA & 8666423 & 272466 & $6.59 \mathrm{~km}$ & 60 m.n.s.m. \\
\hline 021 & PARTICULAR & SAN MIGUEL & 8663834 & 273478 & $6.65 \mathrm{~km}$ & 62 m.n.s.m. \\
\hline 022 & SEDAPAL & SURQUILLO & 8660966 & 279602 & $6.70 \mathrm{~km}$ & 110 m.n.s.m. \\
\hline 023 & SEDAPAL & BELLAVISTA & 8666414 & 272136 & $6.91 \mathrm{~km}$ & 55 m.n.s.m. \\
\hline 024 & SEDAPAL & CARMEN DE LA LEGUA & 8667898 & 272031 & $6.92 \mathrm{~km}$ & 56 m.n.s.m. \\
\hline 025 & PARTICULAR & BELLAVISTA & 8665979 & 272196 & $6.95 \mathrm{~km}$ & 56 m.n.s.m. \\
\hline 026 & SEDAPAL & SAN MIGUEL & 8663108 & 273624 & $6.98 \mathrm{~km}$ & 61 m.n.s.m. \\
\hline 027 & SEDAPAL & LA MOLINA & 8665101 & 285565 & $7.11 \mathrm{~km}$ & 228 m.n.s.m. \\
\hline 028 & SEDAPAL & MAGDALENA & 8661690 & 275023 & $7.12 \mathrm{~km}$ & 63 m.n.s.m. \\
\hline 029 & SEDAPAL & SAN BORJA & 8660909 & 281642 & $7.25 \mathrm{~km}$ & 135 m.n.s.m. \\
\hline 030 & SEDAPAL & LA MOLINA & 8664838 & 285671 & $7.30 \mathrm{~km}$ & 278 m.n.s.m. \\
\hline 031 & SEDAPAL & SAN BORJA & 8661025 & 282127 & $7.33 \mathrm{~km}$ & 143 m.n.s.m. \\
\hline 032 & SEDAPAL & LA MOLINA & 8665166 & 285895 & $7.37 \mathrm{~km}$ & 236 m.n.s.m. \\
\hline 033 & SEDAPAL & SANTA ANITA & 8667143 & 286323 & $7.40 \mathrm{~km}$ & 262 m.n.s.m. \\
\hline 034 & SEDAPAL & SAN MIGUEL & 8665951 & 380767 & $7.42 \mathrm{~km}$ & 50 m.n.s.m. \\
\hline 035 & SEDAPAL & SANTIAGO DE SURCO & 8662239 & 284100 & $7.46 \mathrm{~km}$ & 178 m.n.s.m. \\
\hline 036 & SEDAPAL & SANTIAGO DE SURCO & 8663015 & 284826 & $7.47 \mathrm{~km}$ & 195 m.n.s.m. \\
\hline 037 & SEDAPAL & BELLAVISTA & 8666970 & 380430 & $7.50 \mathrm{~km}$ & 48 m.n.s.m. \\
\hline 038 & SEDAPAL & SANTA ANITA & 8668884 & 286435 & $7.59 \mathrm{~km}$ & 275 m.n.s.m. \\
\hline 039 & SEDAPAL & CALLAO & 8666865 & 271360 & $7.62 \mathrm{~km}$ & 48 m.n.s.m. \\
\hline 040 & SEDAPAL & CARMEN DE LA LEGUA & 8667844 & 271307 & $7.64 \mathrm{~km}$ & 45 m.n.s.m. \\
\hline 041 & SEDAPAL & SANTA ANITA & 8669252 & 286430 & $7.66 \mathrm{~km}$ & 275 m.n.s.m. \\
\hline 042 & SEDAPAL & SAN MIGUEL & 8663847 & 272202 & $7.73 \mathrm{~km}$ & 49 m.n.s.m. \\
\hline 043 & SEDAPAL & LA MOLINA & 8665423 & 286354 & $7.74 \mathrm{~km}$ & 245 m.n.s.m. \\
\hline 044 & SEDAPAL & SANTA ANITA & 8668292 & 286894 & $7.98 \mathrm{~km}$ & 282 m.n.s.m. \\
\hline 045 & SEDAPAL & SANTA ANITA & 8668954 & 286912 & $8.10 \mathrm{~km}$ & 286 m.n.s.m. \\
\hline 046 & SEDAPAL & SURQUILLO & 8669577 & 389683 & $8.11 \mathrm{~km}$ & 113 m.n.s.m. \\
\hline 047 & SEDAPAL & BELLAVISTA & 8666085 & 270729 & $8.36 \mathrm{~km}$ & 40 m.n.s.m. \\
\hline 048 & SEDAPAL & SANTIAGO DE SURCO & 8660238 & 282987 & $8.42 \mathrm{~km}$ & 140 m.n.s.m. \\
\hline 049 & SEDAPAL & SANTA ANITA & 8668451 & 287450 & $8.56 \mathrm{~km}$ & 292 m.n.s.m. \\
\hline 050 & SEDAPAL & CALLAO & 8666550 & 270403 & $8.61 \mathrm{~km}$ & 36 m.n.s.m. \\
\hline 051 & SEDAPAL & SANTA ANITA & 8669124 & 287526 & $8.72 \mathrm{~km}$ & 299 m.n.s.m. \\
\hline 052 & SEDAPAL & AGUSTINO & 8669912 & 8669912 & $8.72 \mathrm{~km}$ & 291 m.n.s.m. \\
\hline 053 & SEDAPAL & ATE & 8667070 & 287684 & $8.76 \mathrm{~km}$ & 208 m.n.s.m. \\
\hline 054 & SEDAPAL & CALLAO & 8667876 & 270171 & $8.78 \mathrm{~km}$ & 31 m.n.s.m. \\
\hline 055 & PARTICULAR & SANTA ANITA & 8668314 & 287730 & $8.82 \mathrm{~km}$ & 299 m.n.s.m. \\
\hline 056 & SEDAPAL & SANTIAGO DE SURCO & 8660087 & 283637 & $8.88 \mathrm{~km}$ & 144 m.n.s.m. \\
\hline 057 & SEDAPAL & LA MOLINA & 8665415 & 287607 & $8.94 \mathrm{~km}$ & 255 m.n.s.m. \\
\hline 058 & SEDAPAL & SANTIAGO DE SURCO & 8659629 & 282990 & $8.96 \mathrm{~km}$ & 131 m.n.s.m. \\
\hline 059 & SEDAPAL & SANTIAGO DE SURCO & 8660580 & 284454 & $8.95 \mathrm{~km}$ & 157 m.n.s.m. \\
\hline 060 & SEDAPAL & CALLAO & 8667990 & 269895 & $9.10 \mathrm{~km}$ & 29 m.n.s.m. \\
\hline 061 & SEDAPAL & BELLAVISTA & 8666144 & 269933 & $9.14 \mathrm{~km}$ & 33 m.n.s.m. \\
\hline 062 & SEDAPAL & SANTIAGO DE SURCO & 8660654 & 284997 & $9.23 \mathrm{~km}$ & 162 m.n.s.m. \\
\hline 063 & SEDAPAL & LA PERLA & 8664794 & 270086 & $9.30 \mathrm{~km}$ & 28 m.n.s.m. \\
\hline 064 & SEDAPAL & CALLAO & 8666478 & 269611 & $9.40 \mathrm{~km}$ & 28 m.n.s.m. \\
\hline 065 & SEDAPAL & SANTIAGO DE SURCO & 8659447 & 283659 & $9.44 \mathrm{~km}$ & 134 m.n.s.m. \\
\hline 066 & SEDAPAL & LA PERLA & 8664254 & 270104 & $9.46 \mathrm{~km}$ & 34 m.n.s.m. \\
\hline 067 & SEDAPAL & SANTIAGO DE SURCO & 8659188 & 283825 & $9.75 \mathrm{~km}$ & 131 m.n.s.m. \\
\hline 068 & SEDAPAL & ATE & 8669174 & 288648 & $9.83 \mathrm{~km}$ & 316 m.n.s.m. \\
\hline 069 & SEDAPAL & SANTIAGO DE SURCO & 8658666 & 283036 & $9.85 \mathrm{~km}$ & 117 m.n.s.m. \\
\hline 070 & SEDAPAL & LA MOLINA & 8665700 & 288666 & $9.92 \mathrm{~km}$ & 267 m.n.s.m. \\
\hline 071 & SEDAPAL & SANTIAGO DE SURCO & 8658186 & 282304 & $10.00 \mathrm{~km}$ & 105 m.n.s.m. \\
\hline 072 & SEDAPAL & LA PERLA & 8665321 & 269200 & $10.00 \mathrm{~km}$ & 23 m.n.s.m. \\
\hline 073 & SEDAPAL & CALLAO & 8667755 & 268882 & $10.00 \mathrm{~km}$ & 20 m.n.s.m. \\
\hline 074 & SEDAPAL & LA MOLINA & 8663358 & 288175 & $10.20 \mathrm{~km}$ & 240 m.n.s.m. \\
\hline 075 & SEDAPAL & SANTIAGO DE SURCO & 8658122 & 283288 & $10.50 \mathrm{~km}$ & 110 m.n.s.m. \\
\hline 076 & SEDAPAL & CALLAO & 8666535 & 268278 & $10.70 \mathrm{~km}$ & 15 m.n.s.m. \\
\hline 077 & SEDAPAL & ATE & 8669589 & 289492 & $10.70 \mathrm{~km}$ & 331 m.n.s.m. \\
\hline 078 & SEDAPAL & LA MOLINA & 8664611 & 289245 & $10.70 \mathrm{~km}$ & 260 m.n.s.m. \\
\hline 079 & SEDAPAL & ATE & 8669105 & 289610 & $10.80 \mathrm{~km}$ & 329 m.n.s.m. \\
\hline 080 & SEDAPAL & CALLAO & 8666406 & 267926 & $11.00 \mathrm{~km}$ & 12 m.n.s.m. \\
\hline 081 & SEDAPAL & ATE & 8670001 & 289861 & $11.20 \mathrm{~km}$ & 331 m.n.s.m. \\
\hline 082 & SEDAPAL & ATE & 8668781 & 290148 & $11.30 \mathrm{~km}$ & 332 m.n.s.m. \\
\hline
\end{tabular}




\begin{tabular}{|c|c|c|c|c|c|c|}
\hline 083 & SEDAPAL & CALLAO & 8667534 & 267588 & $11.40 \mathrm{~km}$ & 8 m.n.s.m. \\
\hline 084 & SEDAPAL & SANTIAGO DE SURCO & 8656692 & 283329 & $11.80 \mathrm{~km}$ & 90 m.n.s.m. \\
\hline 085 & SEDAPAL & SANTIAGO DE SURCO & 8657262 & 284650 & $11.80 \mathrm{~km}$ & 152 m.n.s.m. \\
\hline 086 & SEDAPAL & CALLAO & 8665181 & 267135 & $12.00 \mathrm{~km}$ & 6 m.n.s.m. \\
\hline 087 & SEDAPAL & SURCO & 8655626 & 283167 & $12.70 \mathrm{~km}$ & 74 m.n.s.m. \\
\hline 088 & PARTICULAR & CHORRILLOS & 8655049 & 281379 & $12.80 \mathrm{~km}$ & 60 m.n.s.m. \\
\hline 089 & PARTICULAR & CHORRILLOS & 8655043 & 281196 & $12.80 \mathrm{~km}$ & 60 m.n.s.m. \\
\hline 090 & PARTICULAR & CHORRILLOS & 8655236 & 280914 & $12.80 \mathrm{~km}$ & 59 m.n.s.m. \\
\hline 091 & SEDAPAL & SAN JUAN DE MIRAFLORES & 8656075 & 284365 & $12.80 \mathrm{~km}$ & 93 m.n.s.m. \\
\hline 092 & SEDAPAL & SANTIAGO DE SURCO & 8655686 & 284066 & $13.00 \mathrm{~km}$ & 82 m.n.s.m. \\
\hline 093 & SEDAPAL & ATE & 8670134 & 291998 & $13.30 \mathrm{~km}$ & 373 m.n.s.m. \\
\hline 095 & SEDAPAL & CHORRILLOS & 8653247 & 282462 & $14.80 \mathrm{~km}$ & 46 m.n.s.m. \\
\hline 096 & SEDAPAL & SURCO & 8653544 & 283382 & $14.80 \mathrm{~km}$ & 52 m.n.s.m. \\
\hline 097 & SEDAPAL & ATE & 8670336 & 293660 & $15.00 \mathrm{~km}$ & 399 m.n.s.m. \\
\hline 098 & PARTICULAR & CHORRILLOS & 8652688 & 280483 & $15.00 \mathrm{~km}$ & 38 m.n.s.m. \\
\hline 099 & PARTICULAR & CHORRILLOS & 8651246 & 281882 & $16.60 \mathrm{~km}$ & 15 m.n.s.m. \\
\hline 100 & SEDAPAL & SAN JUAN DE MIRAFLORES & 8651399 & 284800 & $17.30 \mathrm{~km}$ & 71 m.n.s.m. \\
\hline 101 & SEDAPAL & VILLA EL SALVADOR & 8652240 & 287610 & $17.70 \mathrm{~km}$ & 231 m.n.s.m. \\
\hline 102 & SEDAPAL & VILLA MARIA DEL TRIUNFO & 8650588 & 289659 & $18.60 \mathrm{~km}$ & 314 m.n.s.m. \\
\hline 103 & SEDAPAL & CHORRILLOS & 8648444 & 283277 & $19.70 \mathrm{~km}$ & 4 m.n.s.m. \\
\hline 104 & SEDAPAL & VILLA EL SALVADOR & 8649996 & 288678 & $20.10 \mathrm{~km}$ & 171 m.n.s.m. \\
\hline 106 & SEDAPAL & VILLA EL SALVADOR & 8646451 & 288816 & $23.40 \mathrm{~km}$ & 157 m.n.s.m. \\
\hline 107 & PARTICULAR & PACHACÁMAC & 8647116 & 294637 & $25.80 \mathrm{~km}$ & 45 m.n.s.m. \\
\hline 108 & PARTICULAR & LURIN & 8641556 & 297347 & $31.90 \mathrm{~km}$ & 7 m.n.s.m. \\
\hline 109 & SEDAPAL & RIMAC & 8669153 & 278983 & $1.52 \mathrm{~km}$ & 148 m.n.s.m. \\
\hline 110 & SEDAPAL & SAN JUAN DE LURIGANCHO & 12.019 & 77.004 & $4.17 \mathrm{~km}$ & 209 m.n.s.m. \\
\hline 111 & SEDAPAL & SAN MARTIN DE PORRES & 12.015 & 77.057 & $4.48 \mathrm{~km}$ & 99 m.n.s.m. \\
\hline 112 & SEDAPAL & SAN MARTIN DE PORRES & 12.010 & 77.058 & $4.93 \mathrm{~km}$ & 89 m.n.s.m. \\
\hline 113 & SEDAPAL & SAN MARTIN DE PORRES & 12.002 & 77.058 & $5.70 \mathrm{~km}$ & 82 m.n.s.m. \\
\hline 114 & SEDAPAL & SAN MARTIN DE PORRES & 12.031 & 77.082 & $5.80 \mathrm{~km}$ & 71 m.n.s.m. \\
\hline 115 & SEDAPAL & SAN JUAN DE LURIGANCHO & 12.006 & 76.995 & $5.91 \mathrm{~km}$ & 237 m.n.s.m. \\
\hline 116 & SEDAPAL & SAN MARTIN DE PORRES & 12.026 & 77.093 & $7.16 \mathrm{~km}$ & 53 m.n.s.m. \\
\hline 117 & SEDAPAL & CALLAO & 12.034 & 77.097 & $7.32 \mathrm{~km}$ & 50 m.n.s.m. \\
\hline 118 & SEDAPAL & SAN MARTIN DE PORRES & 12.008 & 77.087 & $7.48 \mathrm{~km}$ & 53 m.n.s.m. \\
\hline 119 & SEDAPAL & LOS OLIVOS & 11.993 & 77.076 & $7.68 \mathrm{~km}$ & 56 m.n.s.m. \\
\hline 120 & SEDAPAL & LOS OLIVOS & 11.985 & 77.070 & $7.69 \mathrm{~km}$ & 59 m.n.s.m. \\
\hline 121 & SEDAPAL & SAN JUAN DE LURIGANCHO & 11.976 & 77.017 & $7.85 \mathrm{~km}$ & 284 m.n.s.m. \\
\hline 122 & SEDAPAL & CALLAO & 8671372 & 271689 & $8.17 \mathrm{~km}$ & 39 m.n.s.m. \\
\hline 123 & SEDAPAL & CALLAO & 8671104 & 271394 & $8.31 \mathrm{~km}$ & 36 m.n.s.m. \\
\hline 124 & SEDAPAL & CALLAO & 8671173 & 271361 & $8.37 \mathrm{~km}$ & 37 m.n.s.m. \\
\hline 125 & SEDAPAL & CALLAO & 8670472 & 271038 & $8.40 \mathrm{~km}$ & 37 m.n.s.m. \\
\hline 126 & SEDAPAL & SAN MARTIN DE PORRES & 8671940 & 271668 & $8.46 \mathrm{~km}$ & 40 m.n.s.m. \\
\hline 130 & SEDAPAL & SAN MARTIN DE PORRES & 8674691 & 273081 & $9.18 \mathrm{~km}$ & 49 m.n.s.m. \\
\hline 131 & SEDAPAL & LOS OLIVOS & 8675452 & 273999 & $9.25 \mathrm{~km}$ & 63 m.n.s.m. \\
\hline 132 & SEDAPAL & SAN MARTIN DE PORRES & 8673193 & 271502 & $9.29 \mathrm{~km}$ & 35 m.n.s.m. \\
\hline 133 & SEDAPAL & CALLAO & 8672078 & 270745 & $9.32 \mathrm{~km}$ & 29 m.n.s.m. \\
\hline 134 & SEDAPAL & CALLAO & 8672570 & 271029 & $9.33 \mathrm{~km}$ & 32 m.n.s.m. \\
\hline 135 & SEDAPAL & COMAS & 8676442 & 275735 & $9.38 \mathrm{~km}$ & 77 m.n.s.m. \\
\hline 136 & SEDAPAL & SAN MARTIN DE PORRES & 8674212 & 272109 & $9.48 \mathrm{~km}$ & 36 m.n.s.m. \\
\hline 137 & SEDAPAL & LOS OLIVOS & 8676103 & 274594 & $9.53 \mathrm{~km}$ & 73 m.n.s.m. \\
\hline 138 & SEDAPAL & COMAS & 8676598 & 275336 & $9.65 \mathrm{~km}$ & 79 m.n.s.m. \\
\hline 139 & SEDAPAL & SAN MARTIN DE PORRES & 8675105 & 272667 & $9.76 \mathrm{~km}$ & 51 m.n.s.m. \\
\hline 140 & SEDAPAL & SAN MARTIN DE PORRES & 8673617 & 271029 & $9.92 \mathrm{~km}$ & 31 m.n.s.m. \\
\hline 141 & SEDAPAL & LOS OLIVOS & 8675912 & 273203 & $10.00 \mathrm{~km}$ & 76 m.n.s.m. \\
\hline 142 & SEDAPAL & LOS OLIVOS & 8677036 & 274487 & $10.40 \mathrm{~km}$ & 80 m.n.s.m. \\
\hline 143 & SEDAPAL & SAN MARTIN DE PORRES & 8676387 & 272913 & $10.60 \mathrm{~km}$ & 74 m.n.s.m. \\
\hline 144 & SEDAPAL & LOS OLIVOS & 8676956 & 273667 & $10.70 \mathrm{~km}$ & 74 m.n.s.m. \\
\hline 145 & SEDAPAL & COMAS & 8677857 & 275438 & $10.80 \mathrm{~km}$ & 90 m.n.s.m. \\
\hline 146 & SEDAPAL & COMAS & 8678165 & 276313 & $10.90 \mathrm{~km}$ & 98 m.n.s.m. \\
\hline 147 & SEDAPAL & SAN MARTIN DE PORRES & 8676666 & 272742 & $11.00 \mathrm{~km}$ & 80 m.n.s.m. \\
\hline 148 & SEDAPAL & CALLAO & 8670014 & 268131 & $11.00 \mathrm{~km}$ & 10 m.n.s.m. \\
\hline 149 & SEDAPAL & LOS OLIVOS & 8677634 & 273802 & $11.20 \mathrm{~km}$ & 83 m.n.s.m. \\
\hline 150 & SEDAPAL & CALLAO & 8670009 & 267588 & $11.60 \mathrm{~km}$ & 6 m.n.s.m. \\
\hline 151 & SEDAPAL & COMAS & 8678958 & 276087 & $11.70 \mathrm{~km}$ & 104 m.n.s.m. \\
\hline 152 & SEDAPAL & LOS OLIVOS & 8678446 & 274243 & $11.80 \mathrm{~km}$ & 91 m.n.s.m. \\
\hline 153 & SEDAPAL & COMAS & 8679451 & 276982 & $12.00 \mathrm{~km}$ & 134 m.n.s.m. \\
\hline 154 & SEDAPAL & COMAS & 8679501 & 275632 & $12.30 \mathrm{~km}$ & 112 m.n.s.m. \\
\hline 155 & SEDAPAL & COMAS & 8679600 & 276332 & $12.30 \mathrm{~km}$ & 115 m.n.s.m. \\
\hline 156 & SEDAPAL & LOS OLIVOS & 8681644 & 274103 & $14.80 \mathrm{~km}$ & 112 m.n.s.m. \\
\hline 157 & SEDAPAL & PUENTE PIEDRA & 8681760 & 273889 & $15.00 \mathrm{~km}$ & 140 m.n.s.m. \\
\hline 158 & SEDAPAL & COMAS & 8682830 & 277530 & $15.30 \mathrm{~km}$ & 167 m.n.s.m. \\
\hline 159 & SEDAPAL & COMAS & 8682645 & 277063 & $15.10 \mathrm{~km}$ & 159 m.n.s.m. \\
\hline 160 & SEDAPAL & COMAS & 8682799 & 276335 & $15.40 \mathrm{~km}$ & 153 m.n.s.m. \\
\hline 161 & SEDAPAL & COMAS & 8683611 & 275988 & $16.30 \mathrm{~km}$ & 151 m.n.s.m. \\
\hline 162 & SEDAPAL & CARABAYLLO & 8685930 & 278418 & $18.30 \mathrm{~km}$ & 202 m.n.s.m. \\
\hline 163 & PARTICULAR & CARABAYLLO & 8686592 & 277496 & $19.00 \mathrm{~km}$ & 237 m.n.s.m. \\
\hline 164 & SEDAPAL & CARABAYLLO & 8687058 & 278820 & $19.40 \mathrm{~km}$ & 221 m.n.s.m. \\
\hline 165 & SEDAPAL & CARABAYLLO & 8687180 & 277419 & $19.60 \mathrm{~km}$ & 203 m.n.s.m. \\
\hline 166 & SEDAPAL & CARABAYLLO & 8687986 & 277345 & $20.40 \mathrm{~km}$ & 217 m.n.s.m. \\
\hline 167 & SEDAPAL & $\begin{array}{l}\text { PUENTE PIEDRA } \\
\end{array}$ & 8687828 & 274489 & $20.70 \mathrm{~km}$ & 190 m.n.s.m. \\
\hline 168 & PARTICULAR & CARABAYLLO & 8688307 & 279094 & $20.70 \mathrm{~km}$ & 199 m.n.s.m. \\
\hline 169 & PARTICULAR & CARABAYLLO & 8689163 & 275659 & $21.80 \mathrm{~km}$ & 202 m.n.s.m. \\
\hline
\end{tabular}


devenir Vol. 2, N4, JULIO-DICIEMBRE 2015, Pp. 73-90 - EstudIOS | ISSN 2312-7570

UNIVERSIDAD NACIONAL DE INGENIERÍA, LIMA


Figura 3. Mapeo de las torres de agua y áreas verdes en Lima Metropolitana Fuente: Elaboración propia, 2015.

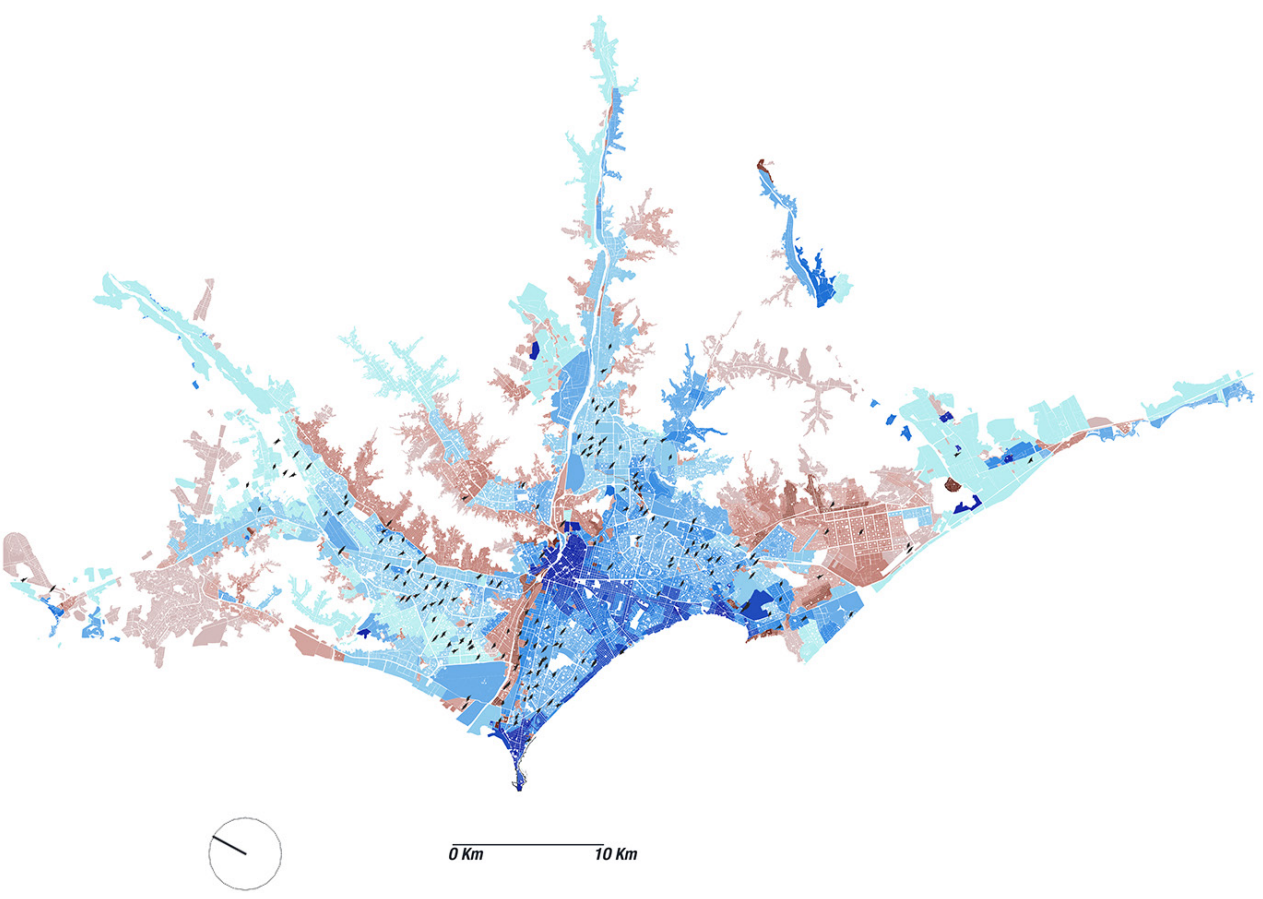

Figura 4. Mapeo de las torres de agua, y el crecimiento formal (azul)e informal (rojo) de Lima Metropolitana Fuente: Elaboración propia sobre la base de información extraída del PLAM 2035, 2015. 


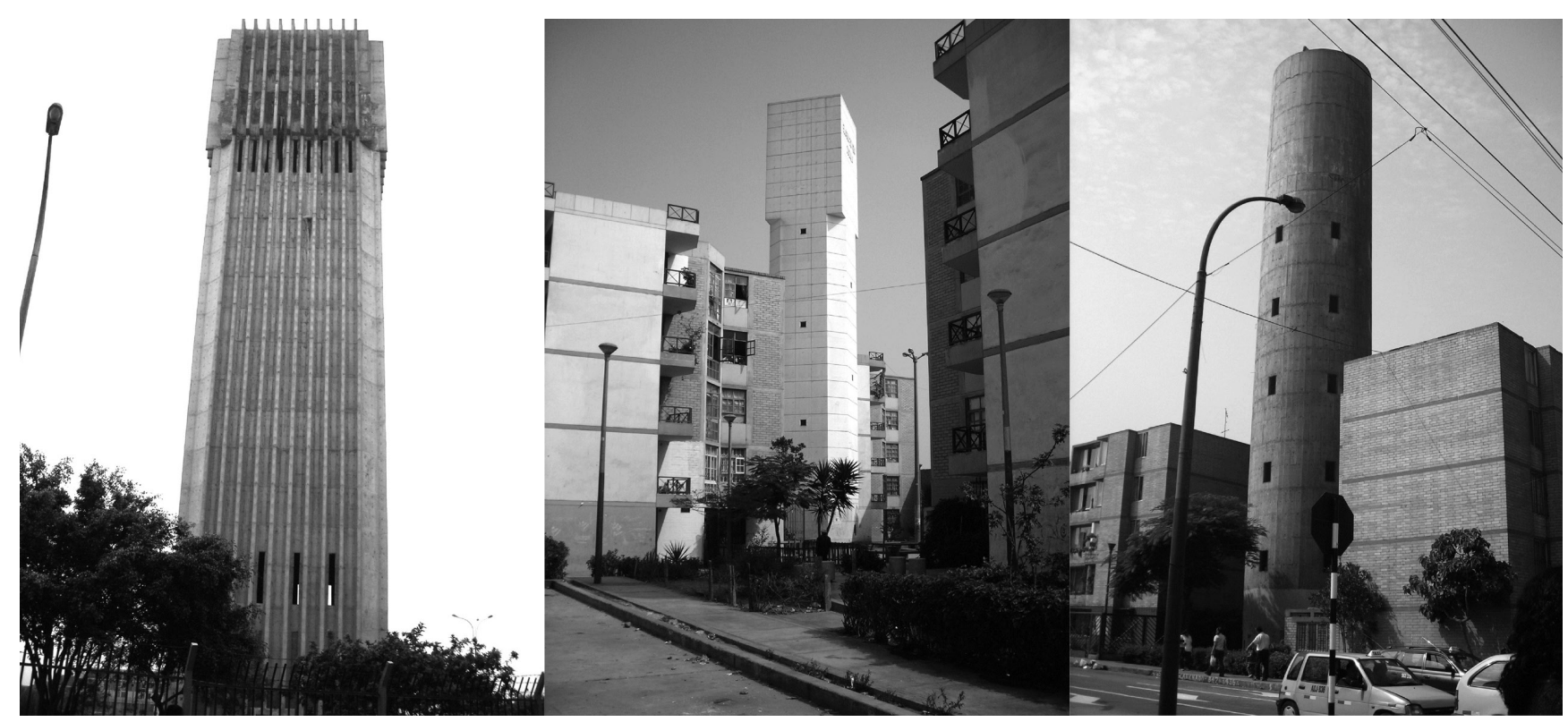

- La ausencia de torres de agua es mayor en los distritos de nivel socioeconómico alto y dentro del triángulo tradicional (Figura 4). Si se considera la virtual ausencia de torres en Miraflores y San Isidro, distritos tradicionales dentro del triángulo Callao, Cercado y Chorrillos, estos son los que presentan menor concentración. Esto se puede deber a muchos factores, entre ellos el carácter formal de la mayor parte del crecimiento dado en estos. Jesús María, Breña, Santa Beatriz y demás tampoco presentan torres de agua.

- Existe una correlación fuerte entre las torres de agua y los pozos (Figura 2). Esta es la conclusión más interesante. El trabajo de campo realizado arrojó que virtualmente toda torre de agua venía acompañada de una bomba conectada a un pozo. La hipótesis era la interdependencia de estos. Al secarse los pozos, con el avanzar de la ciudad y el tiempo, el grado de utilidad de las torres de agua disminuyó. La más clara demostración de ello está en la zona de Surco.

- Existe una correlación directa entre el recorrido del río Surco y la dispersión de las torres de agua (Figura 2). La hipótesis anterior de interdependencia se refuerza si se considera el casi nulo caudal del río Surco y el uso de las torres de agua en ese distrito. En el trabajo de campo no se encontró ninguna torre de agua en ese distrito que estuviese operativa.

- Existe una correlación fuerte entre la población distrital y la cantidad de torres de agua. En San Martín de Porres hay 40 mil habitantes por torre de agua; cabe resaltar que Surco presenta la misma cantidad de torres. En el caso del Callao, hay una por cada 30,300 personas; en el caso de Ate, cada 53,333 habitantes. Sin embargo, resulta notorio que el último también presenta una gran cantidad de reservorios en altura apoyados en las estribaciones. Si se proyectan las curvas de crecimiento, tomando como referencia la torre de agua más antigua estudiada (se estima que fue construida en 1951), se puede inferir incluso que se proyectaba que cada torre de agua sirva a 5 mil personas.

- Existe una correlación fuerte entre la ubicación de las torres de agua y las áreas verdes públicas. En efecto, como se puede ver en la Figura 3, del total de torres de agua registradas, el $72 \%$ se encuentra en un área verde pública. Esto permite inferir que se usaban también para el riego de estas áreas.
Figura 5. Relevamiento de las torres en San Borja y Cercado de Lima Fuente: Elaboración propia, 2008. 
devenir Vol. 2, N4, JULIO-DICIEMBRE 2015, PP. 73-90 - EstudIOS | ISSN 2312-7570

UniverSIDAD NACIONAL DE INGENIERÍA, LIMA

Figura 6. Tipología según volumen y sombras

Fuente: Elaboración propia, 2015.


Figura 7. Tipología según plantas y sombras

Fuente: Elaboración propia, 2015.


醇:

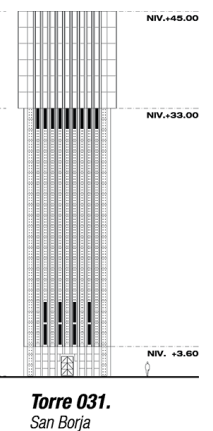

Plantas mostrando la tipologia proyectada.



Elevaciones con sombra.

Figura 8. Tipología según elevación y alturas

Fuente: Elaboración propia, 2015.
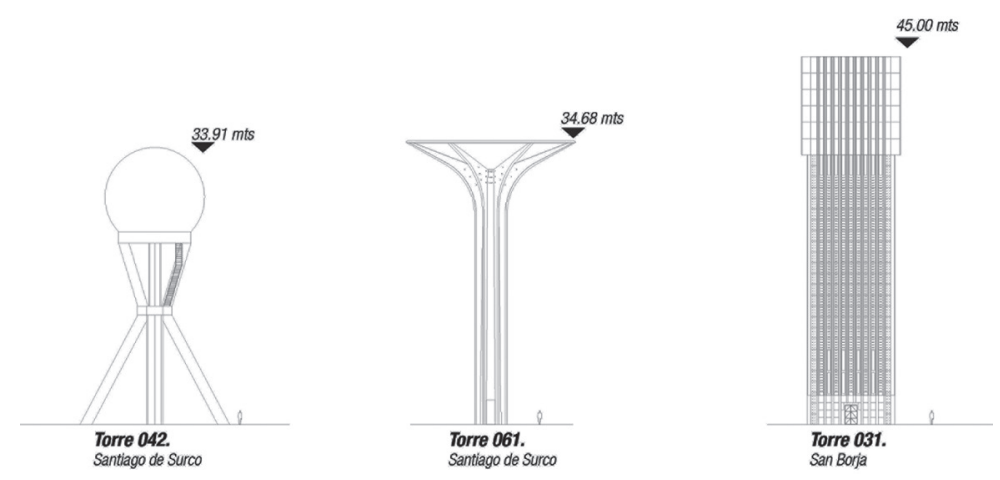
- Existe una correlación fuerte entre el crecimiento formal de Lima Metropolitana y las torres de agua. En efecto, como se puede observar en la Figura 4, si se considera las manchas de crecimiento formal (azul) e informal (rojo), fuera del triángulo tradicional existe una correlación del 96\% de consistencia entre el crecimiento de la Lima planificada y la distribución de torres de agua.

\section{Determinación Patrimonial. Caso Torre UV3}

El enfoque del presente argumento sería inválido como propuesta si no se tuviese el alcance sociológico demostrable. Desde la determinación del problema, que es argumentar una definición de patrimonio utilitario a las torres de agua, se presenta el caso de aceptación, y pérdida de un bien patrimonial y de imaginario urbano.

Previamente se comentó la significación de la Torre de Agua de la Unidad Vecinal $N^{\circ} 3$. Este conjunto habitacional se edificó, desde el gobierno de Bustamante y Rivero hasta el de Odría, con el objetivo de proporcionar vivienda para la clase media baja. El proyecto fue encargado a un equipo que incluyó a Fernando Belaúnde Terry y a Luis Dórich. Menester del tema era dotar de agua a las casi 5,500 personas que la habitarían. La idea de las Unidades Vecinales era retomar la idea de clúster vivencial, que permitiese tener situaciones autónomas e independientes, y facilitase establecer servicios y viviendas para plantear una mejor alternativa de vivienda a la población de Lima, en ese momento ya afectada por la migración campesina. Se data el inicio del proyecto en 1946 y se estima la finalización de la torre de agua en 1951.

En ese momento era, junto a la torre de agua de la Universidad Mayor de San Marcos, una de las estructuras más altas de Lima. Cabe recordar también que la modernidad, tardíamente, estaba llegando. La agrupación Espacio estaba recién en ciernes, y las obras de ensanches, remodelaciones y en general la adopción tardía de un modelo foráneo empezaba. La torre de agua UV3 se alimentaba de los pozos y acuíferos presentes todavía en la zona del fundo. La Av. Colonial y la Av. Universitaria recién se encontraban.

La significancia de esta torre de agua no es solo un tema contextual, es también una cuestión de representatividad. Todas las generaciones que se alimentaban al pie de la torre de agua veían una niñez ajena a ella, tal vez. De acuerdo a la data recogida, el $98 \%$ consideraba a la torre de agua parte de la UV3 y parte de su vivir (Leandro Flores, 2006).

Si retomamos el argumento heideggeriano sobre el Dasein, podríamos aceptar como parte del ente arrojado al contexto y apropiado a la torre de agua. En las mismas recopilaciones verbales, se notaban básicamente tres aspectos:

1. Su altura. Era visible desde el punto más lejano (más de 740 metros) respecto a la ubicación de la misma. El diseño de baja densidad y gran área libre ayudaba a la colección mental de la misma en el imaginario colectivo.

2. Su ubicación. Estaba ubicada en plena Av. Universitaria (era el ingreso general desde ese sentido), al final de una de las zonas de jardín más grandes de la UV3.

3. Su forma. Esta torre de agua tenía la característica de aparente movimiento. Las recopilaciones de testimonios indican que la geometría aportaba ese sentido de esbeltez. La configuración de las vigas formaba una estrella de seis puntas, las cuales se podían ver, como se mencionó, desde lejos, mientras que la base daba apariencia flotante a la estructura. Esto contrastaba con lo sinuoso de la escalera, que adosaba y recorría la envergadura, y remataba en el reservorio achaflanado. El hecho de haber hecho el fuste hueco, aparentemente para reducir costos, lo hacía menos pesado.

Estas tres condiciones demostraban la imagen presencial y la trascendencia de la torre de agua UV3, que se mantuvo igual hasta el 2 de enero de 2008. Dadas las obras de habilitación y ensanche de la Avenida Universitaria, así como la construcción de pasos 
devenir Vol. 2, N4, JULIO-DICIEMBRE 2015, PP. 73-90 - EstudIOS | ISSN 2312-7570

UnIVERSIDAD NACIONAL DE INGENIERÍA, LIMA

Figura 9. Relevamiento fotográfico, enero 2008

Fuente: Elaboración propia, 2008.


diós tanque que miraste desde lo alto a esta Unidad durante tantos años Juana"
Figura 10. Relevamiento fotográfico, enero 2008

Fuente: Elaboración propia, 2008. 




a desnivel, el gobierno municipal, liderado por Luis Castañeda Lossio, dispuso su demolición para poder ensanchar la vía que conectaría con la Universidad Nacional Mayor de San Marcos. Estas obras abarcaban desde la Av. Colonial hasta la Av. Venezuela.

Inicialmente un equipo de ocho personas se encargó de demolerla. Preliminarmente se cerró la zona con pantallas de rafia blanca, que bordeaban la torre de agua.La demolición duró aproximadamente cuatro meses. El punto de interés no es la demolición en sí; no es tampoco la ausencia de un hito dentro de la ciudad. Lo interesante es la identificación del colectivo con un bien aún no oficial.

Como se puede apreciar en la Figura 9, la aceptación del ente como parte del colectivo, en este caso la torre de agua como ente y los vecinos como el colectivo, denota un arraigo e identificación singular con el hecho.

Se puede reconocer en la Figura 09_01 la obsolescencia proyectada por el individuo; en la Figura 09_02 y 09_03, la identificación temporal. En la Figura 09_04 está la proyección sublimada: se está dando incluso identidad al ente.
Figura 11. Relevamiento fotográfico enero 2008

Fuente: Elaboración propia, 2008. 
En la Figura 10_05 se puede ver la cualidad estética proyectada hacia el ente: se lo reconoce como bello. En la Figura 10_06 se observa la pulsión de permanencia, de reconocimiento memorial. En la Figura 10_07 es visible el reconocimiento físico; se le proyecta incluso la cualidad de humano al darle la facultad de observancia. Finalmente, en la Figura 10_08 se le faculta el agradecimiento, la consecuencia de la ausencia y el deseo reflejado.

¿Qué es hacer propio algo? Reconocerlo. Valorarlo para que trascienda y, cuando ya no esté, testimoniarlo. Hacerlo trascendente a través de la memoria colectiva. Este es el caso de un ente patrimonial que nació tan pronto murió.

Finalmente, volviendo a Heidegger, este sostiene que lo único estable en el Dasein, lo único que puede hacer que trascienda, es la búsqueda de la verdad, y la única verdad inmanente es el fin, que es la muerte. Solo aceptando la muerte como único fin, se podrá tener un Dasein completo.

En este caso, por la muerte fue completo, y no fue completo sino hasta su muerte.

\section{Referencias}

Ballart, J. \& Juan-Tresserras, J. (2001). Gestión del patrimonio cultural. Barcelona, España: Ariel.

Cercleaux, A., Merciu, F., \& Peptenatu, D. (2014). Conversion of water towers: An instrument for conserving heritage assets. Recuperado de http://uac.incd.ro/Art/v5n2a01.pdf

Hayakawa, J. (2012). Restauro UNI. Breve antología de textos de restauración del patrimonio monumental edificado. Lima, Perú: Universidad Nacional de Ingenería.

Heidegger, M. (2003). Ser y tiempo (J. E. Rivera, Trad.). Madrid, España:Trotta. (1927)

Heidegger, M. (2009). De la esencia de la verdad. Barcelona, España: Herder.

Kostof, S. (1991). The city shaped. Londres, Reino Unido: Bulfinch Press.

Leandro Flores, I. (2006). Bestiario de las torres de agua. Manuscrito sin publicar

Merriam-Webster, D. (2014). Dictionary Merriam-Webster, 11th edition. Versión En Línea. Massachusetts: EUNA.

Real Academia Española (RAE). (2005). Diccionario de la Real Academia de la Lengua Española. Madrid, España: ESPASA.

Tower. (12 de agosto de 2015). En Online Etimology Dictionary. Recuperado de http://www.etymonline.com

Vázquez Fernández, A. (2014). Tres conceptos de alteridad: Una lectura actitudinal. Daímon. Revista Internacional de Filosofía, 77.

West, G. (7 de julio de 2015). Making sense of the new science of cities. California: TED Talk. 